\title{
Heart failure and the risk of stroke: the Rotterdam Study
}

\author{
V. P. Alberts $\cdot$ M. J. Bos $\cdot$ P. J. Koudstaal $\cdot$ \\ A. Hofman - J. C. M. Witteman - B. H. C. Stricker • \\ M. M. B. Breteler
}

Received: 2 May 2010/ Accepted: 19 October 2010/Published online: 9 November 2010

(C) The Author(s) 2010. This article is published with open access at Springerlink.com

\begin{abstract}
Patients with heart failure used to have an increased risk of stroke, but this may have changed with current treatment regimens. We assessed the association between heart failure and the risk of stroke in a populationbased cohort that was followed since 1990. The study uses the cohort of the Rotterdam Study and is based on 7,546 participants who at baseline (1990-1993) were aged 55 years or over and free from stroke. The associations between heart failure and risk of stroke were assessed using time-dependent Cox proportional hazards models, adjusted for cardiovascular risk factors (smoking, diabetes mellitus, BMI, ankle brachial index, blood pressure, atrial fibrillation, myocardial infarction and relevant medication). At baseline, 233 participants had heart failure. During an average follow-up time of 9.7 years, 1,014 persons developed heart failure, and 827 strokes (470 ischemic, 75 hemorrhagic, 282 unclassified) occurred. The risk of ischemic stroke was more than five-fold increased in the first month after diagnosis of heart failure (age and sex adjusted HR 5.79, 95\% CI 2.15-15.62), but attenuated over time (age and sex adjusted HR 3.50 [95\% CI 1.96-6.25] after 1-6 months and 0.83 [95\% CI $0.53-1.29$ ] after 0.5-6 years). Additional adjustment for cardiovascular risk factors only marginally attenuated these risks. In
\end{abstract}

V. P. Alberts - M. J. Bos - A. Hofman .

J. C. M. Witteman - B. H. C. Stricker - M. M. B. Breteler $(\square)$

Department of Epidemiology, Erasmus MC University Medical

Center, Dr. Molewaterplein 50, PO Box 2040,

3000 CA Rotterdam, The Netherlands

e-mail: m.breteler@erasmusmc.nl

M. J. Bos · P. J. Koudstaal

Department of Neurology, Erasmus MC University Medical

Center, Dr. Molewaterplein 50, PO Box 2040,

3000 CA Rotterdam, The Netherlands conclusion, the risk of ischemic stroke is strongly increased shortly after the diagnosis of heart failure but returns to normal within 6 months after onset of heart failure.

Keywords Stroke $\cdot$ Heart Failure $\cdot$ Elderly $\cdot$ Cohort Study

\section{Introduction}

Almost one out of three persons of 55 year and over will develop heart failure during their remaining lifespan [1-5]. Because of its high incidence, heart failure may have an important impact on the incidence of other diseases, including stroke [6].

Previous studies found that the risk of stroke among heart failure patients is almost 7 times higher than in the general population [7-13]. However, most population-based studies were performed before the 1990s when ACE inhibitors were introduced on a large scale. Current guidelines recommend the use of ACE inhibitors in all patients with symptomatic systolic heart failure [14]. The survival rate of heart failure has improved significantly over the past decades, and also the risk of stroke in heart failure patients was higher in studies performed before 1990 than in more recent ones $[9,15]$. This makes it hard to extrapolate findings from previous population-based studies to the present day; in addition, most previous studies did not adjust for confounders other than age and sex $[8,11,12,16]$. In 2006, Witt et al. found a 17.4-fold increased risk of stroke in the fist 30 days after heart failure diagnosis, which was reduced to 2.9-fold after 5 years [16]. This suggests that the risk of stroke varies with time after the diagnosis of heart failure.

We investigated the association between heart failure and the risk of stroke in a large prospective populationbased cohort study that started in 1990. To compare our 
results with previous studies, which almost invariably focused on the short-term prognosis of heart failure, we also wished to investigate the longer term-prognosis of heart failure and assessed the risk of stroke in various time intervals after the diagnosis of heart failure.

\section{Methods}

Population

The present study is part of the Rotterdam Study, a population-based prospective cohort study on chronic and disabling diseases. All inhabitants of Ommoord, a district in the city of Rotterdam in the Netherlands, who were 55 years or older were invited to participate. Of all 10,275 invited subjects, 7,983 were entered into the study (78\%) [17]. The Medical Ethics Committee of the Erasmus Medical Center approved the study. Baseline examinations, which consisted of a home interview and clinical workup at the research center, were conducted between 1990 and 1993. Subsequently, participants were continuously monitored for major events, including stroke and heart failure, through automated linkage of the study database with files from general practitioners. Information on vital status is obtained regularly from municipal health authorities in Rotterdam. Furthermore, all drug prescriptions dispensed to participants by all pharmacies in the study area are routinely stored in the database. After exclusion of participants who refused informed consent $(n=174)$, participants with missing data $(n=20)$, or who had a stroke before baseline $(n=243), 7,546$ persons were included in this study.

\section{Assessment of heart failure}

Prevalent heart failure at baseline was assessed using a validated score, that was based on the heart failure definition of the European Society of Cardiology [18]. More details of the assessment have been described previously $[1,19]$. Prevalent heart failure cases were obtained through a database containing hospital discharge diagnoses from all hospitals in the Rotterdam area as of January 1, 1991 [20]. Furthermore, all medical records were screened in retrospect for the occurrence of heart failure in most (97\%) participants of the Rotterdam Study. With these three methods, information on the presence of heart failure at baseline was available for all participants.

Cases of incident heart failure were obtained by continuously monitoring participants of the Rotterdam Study for the occurrence of heart failure during follow-up through automated linkage with files from general practitioners. Also, we used verified hospital discharge diagnoses for case finding, gathered from all hospitals in the Rotterdam area. The date of incident heart failure was defined as the day of the first occurrence of symptoms suggestive of heart failure, obtained from the medical records, or the day of receipt of a first prescription for a loop diuretic or an ACEinhibitor indicated for treatment of heart failure, whichever came first.

The diagnosis of heart failure was classified as definite, probable, possible, or unlikely. Only definite and probable cases were considered in the analyses. In accordance with the criteria of the European Society of Cardiology, definite heart failure was defined as a combination of heart failure diagnosed by a medical specialist and the presence of typical symptoms of heart failure, such as breathlessness at rest or during exertion, ankle edema, and pulmonary crepitations, confirmed by objective evidence of cardiac dysfunction (chest X-ray, echocardiography) [18]. Probable heart failure was defined as heart failure diagnosed by a general practitioner, with at least two typical symptoms suggestive of heart failure, and at least 1 of the following: history of cardiovascular disease, response to treatment of heart failure, or objective evidence of cardiac dysfunction, whereas symptoms could not be attributed to another underlying disease.

Two research physicians independently classified all information on potential heart failure events. If there was disagreement, a consensus was reached in a separate session. Finally, a cardiologist verified all probable cases, and all cases in which the two physicians could not reach consensus. If the cardiologist disagreed with the research physicians, the cardiologist's judgment was considered decisive.

\section{Assessment of stroke}

History of stroke at baseline was assessed and verified as described previously [21]. In summary, during the baseline interview, a previous stroke was assessed by asking, "Did you ever suffer from a stroke, diagnosed by a physician?" Medical records of patients who answered yes were checked to verify the diagnosis $[22,23]$. Once subjects are enrolled in the Rotterdam Study, they are monitored continuously for major events through automated linkage of the study database with files from general practitioners and the municipality. In addition, nursing home physicians' files are scrutinized. For reported events, additional information (including brain imaging) is obtained from hospitals. Stroke research physicians reviewed information on all possible strokes; an experienced stroke neurologist (P.J.K.) verified all diagnoses.

Ischemic strokes were diagnosed when a patient had typical symptoms and a CT or MRI within 4 weeks that ruled out other diagnoses or when indirect evidence (deficit 
limited to 1 limb or completely resolved within $72 \mathrm{~h}$, atrial fibrillation in the absence of anticoagulants) pointed at an ischemic nature of the stroke. Hemorrhagic stroke was diagnosed when a relevant hemorrhage was shown on CT or MRI. If a stroke did not match any of these criteria, it was classified as unspecified.

Possible confounders

Blood pressure was measured twice in sitting position on the right arm with a random-zero sphygmomanometer. We used the average of these measurements. A single systolic blood pressure reading was taken at both the left and the right posterior tibial arteries with a Doppler ultrasound $8 \mathrm{MHz}$ transducer while the subject was in the supine position. Ankle-Brachial index was calculated by dividing tibial blood pressure by brachial blood pressure. We considered diabetes mellitus to be present if a random or postload glucose level was more than $11.0 \mathrm{mmol} / \mathrm{l}$ or if a person used antidiabetic medication. Quetelet body mass index (calculated as weight in kilograms divided by the square of height in meters) was assessed at the research center. During the home interview, smoking status (classified as ever or never) and medication use were assessed. Occurrence of myocardial infarction and atrial fibrillation before baseline and during follow-up was assessed and verified as described previously [24, 25].

\section{Statistical analysis}

We calculated hazard ratios with $95 \%$ confidence intervals (95\% CIs) for the associations between heart failure and the risk of stroke, with Cox proportional hazards models. Hazard ratios were calculated for any stroke, ischemic stroke, and hemorrhagic stroke. Furthermore, we assessed whether the association between heart failure and risk of stroke was different in various time intervals (0-30 days, 30 days to 6 months and 6 months to 5 years) after the diagnosis of heart failure. For the 30 days to 6 months period and 6 months to 5 years period, the time between diagnosis of heart failure and start of the period of interest was not counted as person-time at risk. We only used firstever strokes. Subjects were censored at the time of death, end of study or, in case of loss to follow-up, at the date last known to be alive. Follow-up was complete for all participants until January 1, 2005, for $97.1 \%$ of potential person years [26].

All hazard ratios were adjusted for age and sex (model A) and additionally for smoking, diabetes mellitus, BMI, ankle-brachial index, systolic blood pressure, use of antihypertensive drugs, use of antithrombotic drugs and for occurrence of atrial fibrillation and myocardial infarction before baseline and during follow-up (model B) [27-31].
Heart failure, systolic blood pressure, BMI, diabetes mellitus, atrial fibrillation and myocardial infarction were entered as time-dependent covariates. We had $<1 \%$ missings for all variables during the baseline examination except for blood pressure $(n=799)$, ankle brachial index $(n=1,343)$ and BMI $(n=858)$. Mean values were imputed to missing values for blood pressure, ankle brachial index and BMI. Analyses were performed using SPSS 11.0.1 for Windows.

\section{Results}

At baseline, the median age of the study population was 69 years. Of all 7,546 participants, 4,596 were female and 233 had heart failure (baseline characteristics are presented in Table 1). During 73,147 person years of follow-up, 827 first-ever strokes occurred. Of all strokes, 470 were ischemic, 75 hemorrhagic, and 282 could not be specified. Additionally, 1,014 participants developed heart failure.

Participants with heart failure had a higher risk of subsequent stroke than participants without heart failure (Table 2) with no significant differences between men and women $(\mathrm{P}$ interaction $=0.69)$. When adjusted for cardiovascular risk factors, this association disappeared (HR 1.07, 95\% CI 0.86-1.32; model B). For ischemic strokes, the age and sex adjusted hazard ratio was 1.51 (95\% CI 1.15-1.98), which disappeared after adjustment for possible cardiovascular risk factors (HR 1.02, 95\% CI 0.77-1.37). In particular the inclusion of atrial fibrillation in model B caused the association to disappear. Heart failure was not associated with the risk of hemorrhagic stroke (age and sex adjusted HR 1.02, 95\% CI 0.49-2.16). The risk of stroke was highest in the first 30 days after the diagnosis of heart failure (age and sex-adjusted HR 4.27 (95\% CI 1.90-9.60) for stroke, HR 5.79, (95\% CI 2.15

Table 1 Baseline characteristics of the study population $(n=7,546)$

\begin{tabular}{lc}
\hline Characteristic & $\begin{array}{l}\text { Median (interquartile range) } \\
\text { or number (percentage) }\end{array}$ \\
\hline Age (years) & $69.0(62.3-76.7)$ \\
Female sex & $4,596(60.9 \%)$ \\
Ever smoking & $4,649(61.6 \%)$ \\
Diabetes mellitus & $756(10.0 \%)$ \\
Body mass index $\left(\mathrm{kg} / \mathrm{m}^{2}\right)$ & $26.3(24.1-28.0)$ \\
Systolic blood pressure $(\mathrm{mmHg})$ & $139(125-152)$ \\
Ankle brachial index & $1.05(1.00-1.18)$ \\
Antihypertensive drug use & $2,466(32.7 \%)$ \\
ACE-inhibitor use & $466(6.2 \%)$ \\
Antithrombotic drug use & $369(4.9 \%)$ \\
Heart failure (at baseline) & $233(3.1 \%)$ \\
\hline
\end{tabular}


Table 2 Hazard ratios and 95\% CIs for the associations between heart failure and risk of subsequent stroke

\begin{tabular}{lllll}
\hline & & Hazard ratio (95\% CI) & \\
\cline { 3 - 5 } Presence of heart failure & & $\begin{array}{l}\text { All stroke } \\
(n=827)\end{array}$ & $\begin{array}{l}\text { Ischemic stroke } \\
(n=470)\end{array}$ & $\begin{array}{l}\text { Hemorrhagic stroke } \\
(n=75)\end{array}$ \\
\hline No heart failure $(\mathrm{N}=6,299)$ & & 1 (reference) & 1 (reference) & 1 (reference) \\
Heart failure $(\mathrm{N}=1,247)$ & Model A & $1.41(1.15-1.72)$ & $1.51(1.15-1.98)$ & $1.02(0.49-2.16)$ \\
& Model B & $1.07(0.86-1.32)$ & $1.02(0.77-1.37)$ & $0.80(0.37-1.76)$ \\
\hline
\end{tabular}

Model A: Adjusted for age and sex

Model B: Adjusted for age, sex, smoking, diabetes mellitus, BMI, ankle-brachial index, blood pressure, use of antihypertensives, use of antithrombotics, incident and prevalent atrial fibrillation and incident and prevalent myocardial infarction

Table 3 Hazard ratios and 95\% CIs for the association between heart failure and the risk of subsequent stroke and ischemic stroke for various time intervals after the diagnosis of heart failure

\begin{tabular}{llllll}
\hline Outcome & Presence of heart failure & \multicolumn{3}{l}{ Hazard ratio $(95 \% \mathrm{CI})$} & \\
\cline { 3 - 6 } & & & $0-30$ days & 30 days-6 months & 6 months-5 years \\
\hline \multirow{2}{*}{ All stroke } & No heart failure $(\mathrm{N}=6299,749$ strokes) & & 1 (reference) & 1 (reference) & 1 (reference) \\
& Heart failure $(\mathrm{N}=1247,78$ strokes)* & Model A & $4.27(1.90-9.60)$ & $1.94(1.14-3.31)$ & $0.99(0.74-1.33)$ \\
& & Model B & $3.59(1.59-8.10)$ & $1.60(0.93-2.73)$ & $0.78(0.58-1.05)$ \\
Ischemic stroke & No heart failure $(\mathrm{N}=6299,428$ ischemic strokes) & & 1 (reference) & 1 (reference) & 1 (reference) \\
& Heart failure $(\mathrm{N}=1247,42$ ischemic strokes)* & Model A & $5.79(2.15-15.62)$ & $3.50(1.96-6.25)$ & $0.83(0.53-1.29)$ \\
& & Model B & $4.60(1.70-12.49)$ & $2.75(1.53-4.94)$ & $0.58(0.37-0.92)$ \\
\hline
\end{tabular}

Model A: Adjusted for age and sex

Model B: Adjusted for age, sex, smoking, diabetes mellitus, BMI, ankle-brachial index, blood pressure, use of antihypertensives, use of antithrombotics, incident and prevalent atrial fibrillation and incident and prevalent myocardial infarction

* 11 strokes, of which 7 ischemic, occurred between 0 and 30 days after the diagnosis of heart failure, 15 strokes of which 12 ischemic, between 30 days and 6 months, and 52 strokes, of which 23 ischemic, between 6 months and 5 years

15.62) for ischemic stroke (Table 3 ) and decreased significantly with increasing time since first diagnosis of heart failure: $\mathrm{P}$ for interaction between time since diagnosis and heart failure, 0.004 for stroke, and 0.003 for ischemic stroke. The risk remained increased during the first half year after diagnosis of heart failure (Table 3), but was no longer increased thereafter. Adjustments for cardiovascular risk factors (model B) modestly attenuated these hazard ratios, and showed a decreased risk of ischemic stroke after $0.5-6$ years (age and sex adjusted HR $0.58,95 \%$ CI $0.37-0.92$ ).

\section{Discussion}

Men and women with heart failure had a higher risk of stroke than those without heart failure in this populationbased cohort study. The risk of stroke was more than fourfold increased during the month following the diagnosis of heart failure, but had returned to normal by the time people had survived 6 months after the diagnosis of heart failure.

Strengths of our study are its population-based design, the large study population $(N=7,546)$, a mean follow-up of approximately 10 years, and the meticulous ascertainment of heart failure and stroke cases. The follow-up of our cohort started in 1990, so after the introduction of current treatment regimens for heart failure. Our stringent stroke monitoring procedures enabled the ascertainment of stroke cases that had not been referred to a hospital and hence would have been missed had we only relied on hospital records, as is the case in many cohort studies. However, since in these nonhospitalized patients neuroimaging was lacking, $34 \%$ of all strokes could not be subclassified into ischemic or hemorrhagic. Although we adjusted for potential cardiovascular confounders, residual confounding may remain.

Several previous studies reported an increased risk of stroke shortly after the diagnosis of heart failure $[7,8,10$, 16]. Most of these studies had a short follow-up of several months, did not adjust for confounders other than age and sex or were based on hospitalized patients only $[7,8,10]$. In 2006, a study from Rochester followed 630 persons with incident heart failure for a longer period (median follow-up 4.3 years) and reported a 17.4-fold increased risk of stroke in the first 30 days after heart failure diagnosis, which decreased to 2.9-fold after 5 years [16]. In line with the earlier studies we found a 4.3-fold increase for the risk of stroke in the first month after diagnosis of heart failure and 
an almost six-fold increased risk for ischemic stroke in this period, that only slightly attenuated when adjusted for cardiovascular risk factors. However, in our study these increased risks returned to normal within 6 months after onset of heart failure, and the risk of ischemic stroke was even slightly decreased after $0.5-5$ years after the diagnosis of heart failure. This is either a chance finding or may possibly be explained by competing risk or medication effect. A possible explanation for the discrepant findings between our study and the study from Rochester is that our patients developed heart failure after 1991 and most of them were treated with ACE inhibitors, whereas the inclusion period of the Rochester study was stretched over 20 years, from 1979 till 1999, and many persons did not receive current standard treatment.

Heart failure may cause stroke through cardioembolism. Due to the increased risk of stroke in heart failure patients, these persons might benefit from antithrombotic treatment, in particular during the first month after heart failure diagnosis. Currently, a large trial is being undertaken to examine the effect of antithrombotic therapy in patients with heart failure, but this trial assesses the 3-5 year survival while ignoring the first month after heart failure [32, 33]. With an increased risk of stroke after heart failure, and in particular in the first month after onset of heart failure, our results suggest that this study may miss an important window of opportunity to prevent stroke in persons with recent heart failure.

In conclusion, the risk of stroke is strongly increased in the early phase after the diagnosis of heart failure and attenuates over time. Investigating whether a more rigorous treatment of newly diagnosed heart failure patients may result in a decreased incidence of stroke is warranted.

Acknowledgments The Rotterdam Study is supported by Erasmus MC (Erasmus Medical Center Rotterdam), the Erasmus University Rotterdam, the Netherlands Organization for Scientific Research (NWO), the Netherlands Organization for Health Research and Development (ZonMW), the Research Institute for Diseases in the Elderly (RIDE), the Ministry of Education, Culture and Science, and the Ministry of Health, Welfare and Sports.

Open Access This article is distributed under the terms of the Creative Commons Attribution Noncommercial License which permits any noncommercial use, distribution, and reproduction in any medium, provided the original author(s) and source are credited.

\section{References}

1. Bleumink GS, Knetsch AM, Sturkenboom MC, et al. Quantifying the heart failure epidemic: prevalence, incidence rate, lifetime risk and prognosis of heart failure the Rotterdam Study. Eur Heart J. 2004;25:1614-9.
2. Bonneux LG, Huisman CC, de Beer JA. Mortality in 272 European regions, 2002-2004. An update. Eur J Epidemiol. 25:77-85.

3. Thelle DS. The dynamics of cardiovascular epidemiology. Eur J Epidemiol. 2009;24:725-6.

4. Stolk RP, Hutter I, Wittek RP. Population ageing research: a family of disciplines. Eur J Epidemiol. 2009;24:715-8.

5. Chimonas T, Fanouraki I, Liberopoulos EN, Chimonas E, Elisaf M. Diverging trends in cardiovascular morbidity and mortality in a low risk population. Eur J Epidemiol. 2009;24:415-23.

6. Tasevska-Dinevska G, Kennedy LM, Nilsson PM, Willenheimer $\mathrm{R}$. Gender aspects on heart failure incidence and mortality in a middle-aged, urban, community-based population sample: the Malmo preventive project. Eur J Epidemiol. 2009;24:249-57.

7. Szummer KE, Solomon SD, Velazquez EJ, et al. Heart failure on admission and the risk of stroke following acute myocardial infarction: the VALIANT registry. Eur Heart J. 2005;26:2114-9.

8. Wolf PA, Kannel WB, McNamara PM. Occult impaired cardiac function, congestive heart failure, and risk of thrombotic stroke: the Framingham Study. Neurology. 1970;20:373.

9. Witt BJ, Gami AS, Ballman KV, et al. The incidence of ischemic stroke in chronic heart failure: a meta-analysis. J Card Fail. 2007;13:489-96.

10. Mahaffey KW, Harrington RA, Simoons ML, et al. Stroke in patients with acute coronary syndromes: incidence and outcomes in the platelet glycoprotein IIb/IIIa in unstable angina. Receptor suppression using integrilin therapy (PURSUIT) trial. The PURSUIT Investigators. Circulation. 1999;99:2371-7.

11. Katz SD, Marantz PR, Biasucci L, et al. Low incidence of stroke in ambulatory patients with heart failure: a prospective study. Am Heart J. 1993;126:141-6.

12. Wolf PA, Abbott RD, Kannel WB. Atrial fibrillation as an independent risk factor for stroke: the Framingham Study. Stroke. 1991;22:983-8.

13. Gulsvik AK, Thelle DS, Mowe M, Wyller TB. Increased mortality in the slim elderly: a 42 years follow-up study in a general population. Eur J Epidemiol. 2009;24:683-90.

14. Dickstein K, Cohen-Solal A, Filippatos G, et al. ESC Guidelines for the diagnosis and treatment of acute and chronic heart failure 2008: the task force for the diagnosis and treatment of acute and chronic heart failure 2008 of the European Society of Cardiology. Developed in collaboration with the Heart Failure Association of the ESC (HFA) and endorsed by the European Society of Intensive Care Medicine (ESICM). Eur Heart J. 2008;29: 2388-442.

15. Levy D, Kenchaiah S, Larson MG, et al. Long-term trends in the incidence of and survival with heart failure. $\mathrm{N}$ Engl $\mathrm{J}$ Med. 2002;347:1397-402.

16. Witt BJ, Brown RD Jr, Jacobsen SJ, et al. Ischemic stroke after heart failure: a community-based study. Am Heart J. 2006;152: 102-9.

17. Hofman A, Breteler MM, van Duijn CM, et al. The Rotterdam Study: 2010 objectives and design update. Eur J Epidemiol. 2009; 24:553-72.

18. Remme WJ, Swedberg K. Comprehensive guidelines for the diagnosis and treatment of chronic heart failure Task force for the diagnosis and treatment of chronic heart failure of the European Society of Cardiology. Eur J Heart Fail. 2002;4:11-22.

19. Mosterd A, Hoes AW, de Bruyne MC, et al. Prevalence of heart failure and left ventricular dysfunction in the general population; the Rotterdam Study. Eur Heart J. 1999;20:447-55.

20. Merry AH, Boer JM, Schouten LJ, et al. Validity of coronary heart diseases and heart failure based on hospital discharge and mortality data in the Netherlands using the cardiovascular registry Maastricht cohort study. Eur J Epidemiol. 2009;24:237-47.

21. Hollander M, Bots ML, Del Sol AI, et al. Carotid plaques increase the risk of stroke and subtypes of cerebral infarction in 
asymptomatic elderly: the Rotterdam study. Circulation. 2002; 105:2872-7.

22. Bots ML, Looman SJ, Koudstaal PJ, Hofman A, Hoes AW, Grobbee DE. Prevalence of stroke in the general population the Rotterdam Study. Stroke. 1996;27:1499-501.

23. Bots ML, van der Wilk EC, Koudstaal PJ, Hofman A, Grobbee DE. Transient neurological attacks in the general population. Prevalence, risk factors, and clinical relevance. Stroke. 1997;28: 768-73.

24. Heeringa J, van der Kuip DA, Hofman A, et al. Prevalence, incidence and lifetime risk of atrial fibrillation: the Rotterdam study. Eur Heart J. 2006;27:949-53.

25. Ikram MA, Hollander M, Bos MJ, et al. Unrecognized myocardial infarction and the risk of stroke: the Rotterdam Study. Neurology. 2006;67:1635-9.

26. Clark TG, Altman DG, De Stavola BL. Quantification of the completeness of follow-up. Lancet. 2002;359:1309-10.

27. Smith JG, Platonov PG, Hedblad B, Engstrom G, Melander O. Atrial fibrillation in the Malmo diet and cancer study: a study of occurrence, risk factors and diagnostic validity. Eur J Epidemiol. 25:95-102.

28. Heidemann C, Boeing H, Pischon T, Nothlings U, Joost HG, Schulze MB. Association of a diabetes risk score with risk of myocardial infarction, stroke, specific types of cancer, and mortality: a prospective study in the European Prospective Investigation into Cancer and Nutrition (EPIC)-Potsdam cohort. Eur J Epidemiol. 2009;24:281-8.

29. Spencer EA, Pirie KL, Stevens RJ, et al. Diabetes and modifiable risk factors for cardiovascular disease: the prospective million women study. Eur J Epidemiol. 2008;23:793-9.

30. Prugger C, Wellmann J, Heidrich J, Brand-Herrmann SM, Keil U. Cardiovascular risk factors and mortality in patients with coronary heart disease. Eur J Epidemiol. 2008;23:731-7.

31. Romon I, Jougla E, Balkau B, Fagot-Campagna A. The burden of diabetes-related mortality in France in 2002: an analysis using both underlying and multiple causes of death. Eur J Epidemiol. 2008;23:327-34.

32. Freudenberger RS, Schumaecker MM, Homma S. What is the appropriate approach to prevention of thromboembolism in heart failure? Thromb Haemost. 103:489-95.

33. Pullicino P, Thompson JL, Barton B, Levin B, Graham S, Freudenberger RS. Warfarin versus aspirin in patients with reduced cardiac ejection fraction (WARCEF): rationale, objectives, and design. J Card Fail. 2006;12:39-46. 